\title{
IFITMI is a Novel, Highly Sensitive Marker for Endometriotic Stromal Cells in Ovarian and Extragenital Endometriosis
}

\author{
Hui Sun, MD', Shinya Fukuda, MD, PhD', Tetsuya Hirata, MD, PhD'D, \\ Tomoko Arakawa, MD', Suke Ma, MD', Kazuaki Neriishi, MD', Yu Wang, MD, PhD', \\ Arisa Takeuchi, MD', Ai Saeki, MD², Miyuki Harada, MD, PhD', Yasushi Hirota, MD, PhD', \\ Takashi Matsumoto, MD², Kaori Koga, MD, PhD', Osamu Wada-Hiraike, MD, PhD', \\ Masatoshi Kurihara, MD, PhD', Tomoyuki Fujii, MD, PhD', \\ and Yutaka Osuga, MD, PhD'
}

\begin{abstract}
When the presence of endometriotic lesions are not evident by hematoxylin and eosin staining, CDIO is used to highlight and confirm the presence of endometriotic stroma. However, CDIO is not specific only to the endometrial stroma but is also expressed in many other cells. Recently, interferon-inducible transmembrane protein I (IFITMI) was reported as a highly specific immunohistochemical marker of normal endometrial stroma and endometrial stromal neoplasm. In this study, we examined the expression of IFITMI and CDI0 in 18 cases of ovarian endometriosis and 44 cases of extragenital endometriosis. Among the 62 patients, 62 (I00.0\%) were positive for IFITMI and 60 (96.8\%) for CDI0, and CDIO was negative in 2 cases that were positive for IFITMI. Additionally, we found that IFITMI sensitivity was unaffected by the presence or absence of hormonal therapy. To the best of our knowledge, this represents the first demonstration of IFITMI as a highly sensitive stromal marker of ovarian and extragenital endometriosis.
\end{abstract}

\section{Keywords}

endometriosis, IFITMI, stromal marker, CDIO

\section{Introduction}

Endometriosis is a common benign gynecological disease affecting $\sim 10 \%$ of reproductive-aged women. ${ }^{1}$ Endometriosis commonly forms lesions, such as peritoneal, ovarian, and deepfiltrating endometriosis, and less commonly involves the bowel, bladder, or rarely distant sites from the uterus, such as the navel ${ }^{2}$ and lung. ${ }^{3}$ Endometriosis is pathologically diagnosed by the presence of endometrium-like tissues outside the uterus. When the presence of the endometrium is not evident by hematoxylin and eosin staining, CD10 is used to highlight and confirm the presence of endometriotic stroma in cases where diagnosis is difficult. ${ }^{4-7}$ In cases of extragenital endometriosis or microscopic endometriosis lesions, pathological diagnosis can be challenging because endometriotic stroma and glands represent only a minor component of fibrotic endometriotic lesions. ${ }^{8}$ However, CD10 is not specific only to the endometrial stroma but is also expressed in many other cells, including vascular endothelial cells, uterine fibroids, and leiomyosarcoma. ${ }^{5,9}$ Therefore, for better accuracy of diagnosis, development of a better indicator of endometriotic stroma would be beneficial.
Recent studies indicate interferon-inducible transmembrane protein 1 (IFITM1) as a highly specific immunohistochemical marker of normal endometrial stroma and endometrial stromal neoplasms. ${ }^{10}$ Therefore, we hypothesized that IFITM1 might be useful as a novel stromal marker of endometriosis and extragenital endometriosis; however, there are no reports on its status in endometriosis. Here, we comprehensively analyzed IFITM1 expression in endometriosis and extragenital endometriosis.

\footnotetext{
I Department of Obstetrics and Gynecology, University of Tokyo, Tokyo, Japan

2 Department of Gynecology, Osaka Central Hospital, Osaka, Japan

${ }^{3}$ Division of Thoracic Surgery, Pneumothorax Research Center, Nissan Tamagawa Hospital, Tokyo, Japan
}

\section{Corresponding Author:}

Tetsuya Hirata, Department of Obstetrics and Gynecology, Faculty of Medicine, University of Tokyo, 7-3-I Hongo, Bunkyo-ku, Tokyo 8655, Japan. Email: thira-tky@umin.ac.jp 


\section{Methods}

\section{Patients and Samples}

The experimental procedures were approved by the institutional review board of the University of Tokyo (registration no. \#11442). All patients who underwent surgical removal of ovarian endometrioma, as well as bowel, bladder, inguinal, abdominal-wall, and thoracic endometrioses, and diagnosed at the University of Tokyo Hospital, Nissan Tamagawa Hospital, and Osaka Chuo Hospital, were enrolled in this study and provided written informed consent. The diagnosis of endometriosis and extragenital endometriosis was performed through histologic examination performed by certified pathologists. Available hematoxylin and eosin-stained slides were reviewed, and the diagnosis was confirmed in each case. We enrolled 62 patients after reviewing the medical records and recorded patient age at the time of the operation, history of pregnancy, parturition, and preoperative hormonal therapy.

\section{Immunohistochemistry}

Paraffin-embedded specimens were sliced to $5-\mu \mathrm{m}$ thickness, and the sections were placed on slides, deparaffinized, and rehydrated. Antigens were retrieved using an antigenretrieval reagent (Immunosaver; Nissin EM, Tokyo, Japan) at $98^{\circ} \mathrm{C}$, and endogenous peroxidase was blocked by incubation for 5 minutes with a solution of $0.3 \%$ hydrogen peroxidase. Immunohistochemical tissue labeling for IFITM1 (1:500; HPA004810; Sigma-Aldrich, St Louis, Missouri), CD10 (1:1; IR468; Agilent Technologies, Santa Clara, California), estrogen receptor (ER; 1:1; IR084; Agilent Technologies), progesterone receptor (PR; 1:1000; \#8757; Cell Signaling Technology, Tokyo, Japan), and paired-box gene 8 (PAX8; 1:1000; 10336-1-AP; Proteintech, Rosemont, Illinois) was performed using the Dako Envision FLEX+ system (Agilent Technologies). After blocking with a nonspecific staining blocking reagent (Agilent Technologies), the sections were incubated with each specific antibody or rabbit immunoglobulin $\mathrm{G}$ for overnight at $4^{\circ} \mathrm{C}$ and then incubated with peroxidaseconjugated secondary antibody for 30 minutes. Staining was detected using diaminobenzidine chromogen, and all sections were counterstained with hematoxylin and evaluated under a light microscope.

\section{Statistical Analysis}

Statistical analysis was performed using SPSS (v23; IBM Corp, Armonk, New York). Continuous data were reported as mean and standard deviation. Categorical data were analyzed using $\chi^{2}$ tests and Fisher exact tests and represented as counts and percentages. Results were considered statistically significant at a $P<.05$.

\section{Results}

\section{Patient Characteristics}

Table 1 shows the characteristics of patients with ovarian endometrioma and extragenital endometriosis. Eighteen samples of ovarian endometriosis and 44 samples of extragenital endometriosis were used in this study and included 19 intestinal, 8 bladder, 3 ureteral, 3 inguinal, 1 abdominal wall, and 10 diaphragmatic endometrioses. The mean age was $37.6 \pm 6.8$ years for ovarian endometriosis and $38.0 \pm 5.7$ years for extragenital endometriosis. For ovarian endometrioma, none of the patients received hormonal therapy before surgery. Additionally, among the 44 cases of extragenital endometriosis, 21 patients did not undergo preoperative hormone therapy while 16,5 , and 2 patients used a gonadotropin-releasing hormone $(\mathrm{GnRH})$ agonist, dienogest, and oral contraceptives, respectively.

\section{Ovarian Endometrioma}

Both IFITM1 and CD10 were positive in all 18 samples of ovarian endometrioma (Table 2). IFITM1 was localized in the stromal cells just beneath the endometriotic epithelial cells, with a staining pattern that was membranous and cytoplasmic (Figure 1B and D) and similar to the CD10-expression pattern (Figure $1 \mathrm{C}$ and $\mathrm{E}$ ).

\section{Extragenital Endometriosis}

We examined 44 samples of extragenital endometriosis, which included 19 intestine endometrioses, 8 bladder endometrioses, 3 ureteral endometrioses, 3 inguinal endometrioses, 1 abdominal wall endometriosis, and 10 diaphragmatic endometrioses. All extragenital endometrioses were immunoreactive for IFITM1, whereas $42(95.5 \%)$ samples were positive for CD10 (Table 2). Interferon-inducible transmembrane protein 1 was localized in the endometriotic stromal cells, with a staining pattern that was membranous and cytoplasmic (Figure 2A and C) and similar to the CD10 expression pattern (Figure 2B and D). Additionally, we observed weak expression of IFITM1 on the luminal membrane of epithelial cells.

Among the 19 patients with intestine endometriosis, IFITM1 was positive in all cases but CD10 in only 17 cases (Figure 3B and C; Table 2), with the $2 \mathrm{CD} 10$-negative cases involving rectal endometriosis and ileal endometriosis. Additionally, staining for both IFITM1 and CD10 was positive in bladder (Figure 3E and F), ureteral (Figure 3H and $\mathrm{I}$ ), inguinal (Figure $3 \mathrm{~K}$ and $\mathrm{L}$ ), abdominal wall (Figure $3 \mathrm{~N}$ and $\mathrm{O}$ ), and diaphragmatic endometrioses (Figure $3 \mathrm{Q}$ and $\mathrm{R}$; Table 2).

We subsequently examined the 2 cases of negative CD10 expression (Figure 4B and $\mathrm{H}$ ) in detail, finding that endometriotic stromal cells were positive for IFITM1 in both cases (Figure 4C and I). To confirm this, we verified that these endometriotic lesions were also positive for ER (Figure 4D and J), PR (Figure 4E and K), and PAX8 (Figure 4F and L). 


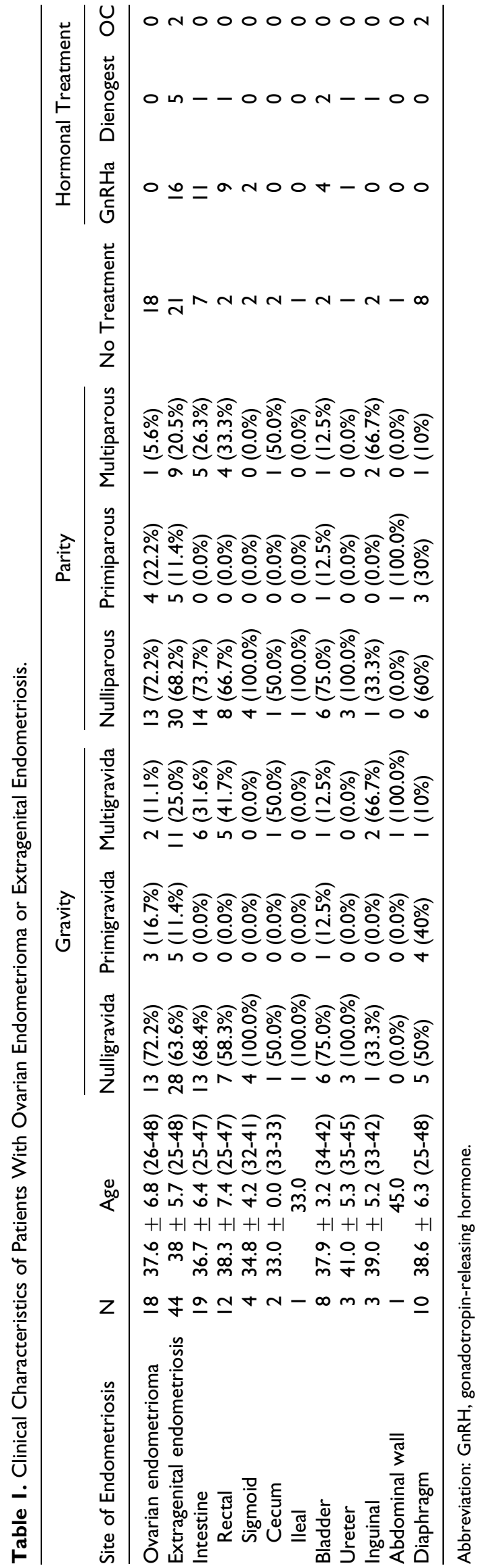

Table 2. The Expression of IFITMI and CDIO in Ovarian Endometrioma or Extragenital Endometriosis.

\begin{tabular}{|c|c|c|c|}
\hline & & IFITMI & CDIO \\
\hline Site of Endometriosis & $\mathrm{N}$ & $\begin{array}{c}\text { Positive/Total } \\
\text { Cases (\%) }\end{array}$ & $\begin{array}{c}\text { Positive/Total } \\
\text { Cases (\%) }\end{array}$ \\
\hline Ovarian endometrioma & 18 & $18 / 18(100.0 \%)$ & $18 / 18(100.0 \%)$ \\
\hline Extragenital endometriosis & 44 & $44 / 44(100.0 \%)$ & $42 / 44(95.5 \%)$ \\
\hline Intestine endometriosis & 19 & $19 / 19(100.0 \%)$ & $17 / 19$ (89.5\%) \\
\hline Rectal & 12 & $12 / 12(100.0 \%)$ & $11 / 12$ (9l.7\%) \\
\hline Sigmoid & 4 & $4 / 4(100.0 \%)$ & $4 / 4(100.0 \%)$ \\
\hline Cecum & 2 & $2 / 2(100.0 \%)$ & $2 / 2(100.0 \%)$ \\
\hline lleal & I & $\mathrm{I} / \mathrm{I}(100.0 \%)$ & $0 / 1(0.0 \%)$ \\
\hline Bladder endometriosis & 8 & $8 / 8(100.0 \%)$ & $8 / 8(100.0 \%)$ \\
\hline Ureteral endometriosis & 3 & $3 / 3(100.0 \%)$ & $3 / 3(100.0 \%)$ \\
\hline Inguinal endometriosis & 3 & $3 / 3(100.0 \%)$ & $3 / 3(100.0 \%)$ \\
\hline Abdominal wall & I & $\mathrm{I} / \mathrm{I}(100.0 \%)$ & $\mathrm{I} / \mathrm{I}(100.0 \%)$ \\
\hline Diaphragm endometriosis & 10 & $10 / 10(100.0 \%)$ & $10 / 10(100.0 \%)$ \\
\hline
\end{tabular}

Abbreviation: IFITMI, Interferon-inducible transmembrane protein I.

\section{The Effect of Hormonal Treatment on IFITMI and $C D / 0$ Expression in Endometriosis}

The positive staining rates of IFITM1 and CD10 were compared between groups with and without hormonal therapy. Among the 62 patients, 23 underwent preoperative hormonal therapy and 39 did not. As shown in Table 3, no significant difference was observed in frequency of positive staining for IFITM1 and CD10.

\section{Discussion}

In this study, we examined IFITM1 and CD10 expression in 18 cases of ovarian endometriosis and 44 cases of extragenital endometriosis. Among the 62 patients, all $(100.0 \%)$ were positive for IFITM1 and $60(96.8 \%)$ for CD10, with 2 cases in which CD10 was negative ultimately determined as positive for IFITM1, ER, PR, and PAX8. Additionally, we found that IFITM1 sensitivity was unaffected by the presence or absence of hormonal therapy. To the best of our knowledge, this represents the first demonstration of IFITM1 as a highly sensitive stromal marker of ovarian and extragenital endometriosis.

To date, CD10 has been used as a useful marker for endometrial stroma and endometriotic stroma because it strongly highlights endometrial or endometriotic stromal cells, which may not have been apparent according to hematoxylin and eosin staining. ${ }^{4-7}$ However, CD10 expression is not specific to endometrial stromal cells and is found in many other cell populations, including hematopoietic cells, renal tubular epithelium, and smooth-muscle cells. ${ }^{5,9}$ Additionally, $20 \%$ to $30 \%$ of benign and malignant smooth-muscle tumors might express CD10, thereby decreasing its specificity. ${ }^{11,12}$ Because extragenital endometriosis can develop in various sites other than those that are common, novel and specific markers for endometrial stroma are required to overcome limitations associated with current immunohistochemical markers. 


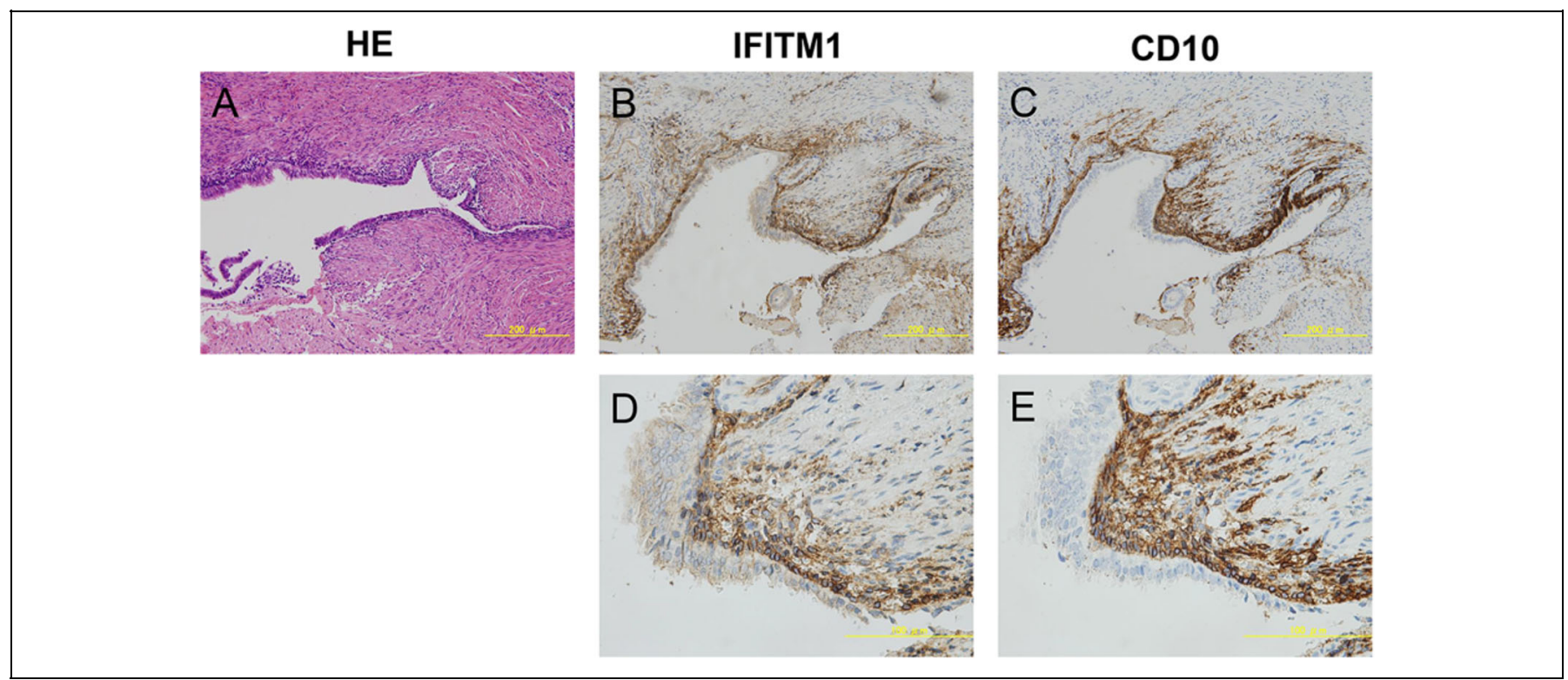

Figure I. Interferon-inducible transmembrane protein I (IFITMI) and CDIO expression in ovarian endometrioma. (A) H\&E and (B and D) IFITMI staining in ovarian endometrioma. IFITMI was localized in the stromal cells just beneath (B), the endometriotic epithelial cells and with a staining pattern that was (D) membranous and cytoplasmic. (C and E) CDIO was localized in the stromal cells of endometrial tissue. Magnification, $\times 200(A-C)$ and $\times 600(D$ and $E)$.

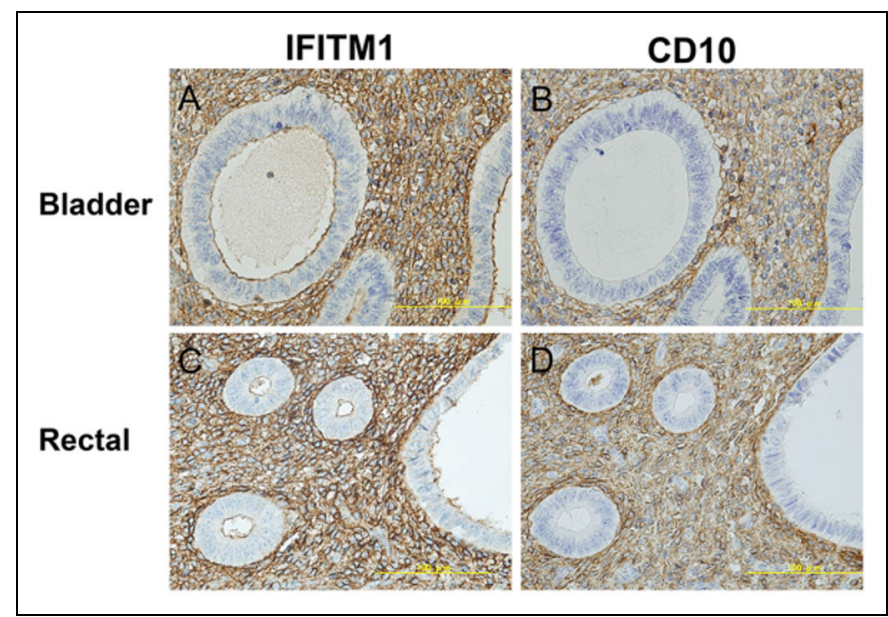

Figure 2. Interferon-inducible transmembrane protein I (IFITMI) and CDIO localization in bladder and rectal endometriosis. $A$ and $C$, IFITMI was localized in endometriotic stromal cells and weakly expressed on the luminal membrane of epithelial cells. B and D, CDIO was localized in endometriotic stromal cells. The staining patterns of IFITMI and CDIO were membranous and cytoplasmic. Magnification, $\times 600$.

Recently, IFITM1 was identified by bioinformatics approaches from a public database of protein expression profiles as an efficacious marker of endometrial stromal-specific molecules. ${ }^{10}$ Interferon-inducible transmembrane protein 1 is a highly specific marker of endometrial stromal differentiation across the spectrum of proliferative endometrium to metastatic endometrial stromal sarcoma. ${ }^{10,13,14}$ Additionally, IFITM1 is expressed on adenomyotic stromal cells and represents a more accurate marker than $\mathrm{CD} 10$ when evaluating the involvement of adenomyosis in endometrioid adenocarcinoma. ${ }^{13}$ Moreover, IFITM1 exhibits similar sensitivity and higher specificity as a marker of endometrial stromal differentiation as compared with CD10. ${ }^{13,14}$ In the present study, we compared the sensitivities of IFITM1 and CD10 for ovarian endometrioma and extragenital endometriosis. Remarkably, IFITM1 was positive in all cases of ovarian and extragenital endometriosis, whereas CD10 was positive in only $96.8 \%$ of cases. This agrees with previous reports showing that $\mathrm{CD} 10$ sensitivity for endometrial stromal cells ranges from $75 \%$ to $100 \%{ }^{5,11,15-17}$ In particular, the present results showed that IFITM1 was expressed in 2 cases of CD10-negative expression, where ER and PR expressions were positive, as was PAX8, which we previously reported as an epithelial marker. These results conclusively identified these lesions as endometriosis and confirmed IFITM1 as a sensitive endometriotic stromal marker comparable or superior to CD10.

In this study, we were unable to clarify IFITM1 specificity because we used only samples diagnosed as endometriosis. However, previous studies of endometrial stroma and endometrial stromal neoplasm showed that IFITM1 is more specific to the endometrial stroma than CD10. ${ }^{13,14}$ Based on these findings, it is likely that IFITM1 specificity might also be high in the endometriotic stroma.

Furthermore, we confirmed that IFITM1 functions as a stromal marker for endometriosis in patients undergoing hormone therapy, such as GnRH agonists and dienogest. When surgery is planned for extragenital endometriosis, preoperative hormone therapy is often used to reduce the size of the lesion or control the condition. Therefore, it is important to verify that these therapies do not alter the expression of endometriosis markers (eg, IFITM1 and CD10). 


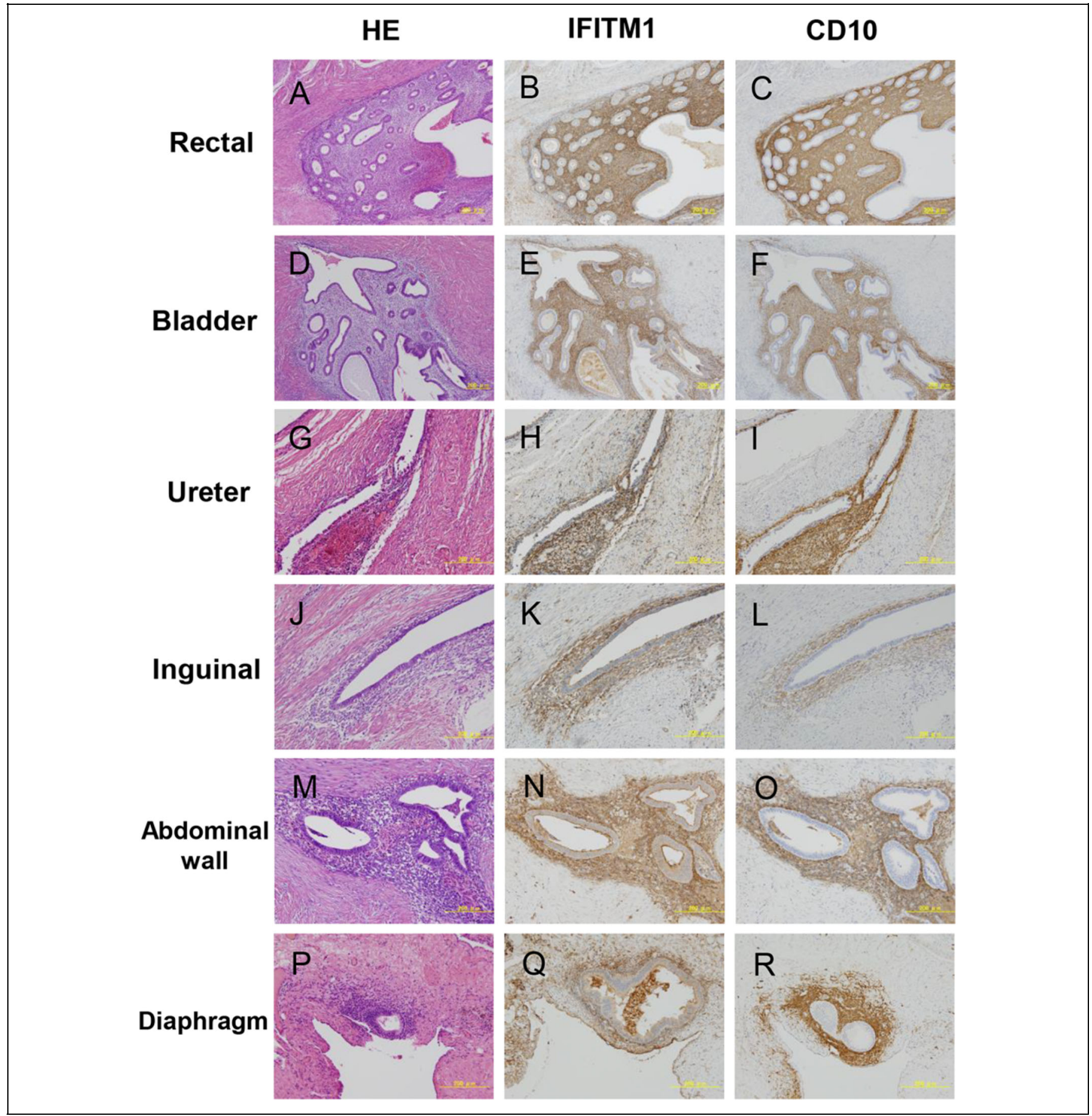

Figure 3. Interferon-inducible transmembrane protein I (IFITMI) and CDIO expression in extragenital endometriosis. (A, D, G, J, M, and P) $H \& E,(B, E, H, K, N$, and Q) IFITMI, and (C, F, I, L, O, and R) CDIO staining in extragenital endometrial tissue. Interferon-inducible transmembrane protein I and CDIO staining was strong and diffuse in the stroma of extragenital endometrial tissue. A-C, Rectal endometriosis, $\mathrm{D}-\mathrm{F}$, bladder endometriosis, G-I, ureteral endometriosis, J-L, inguinal endometriosis, M-O, abdominal-wall endometriosis, and P-R, diaphragmatic endometriosis. Magnification, $\times 100(\mathrm{~A}-\mathrm{F})$ and $\times 200(\mathrm{H}-\mathrm{R})$.

Interferon-inducible transmembrane protein 1 is a member of the IFITM family of proteins ${ }^{18}$ that are strongly induced by type I and type II interferons. ${ }^{19,20}$ Interferon-inducible transmembrane protein 1 is also expressed in lymphoid nongerminal centers, endothelial cells, and epididymal epithelial cells ${ }^{10}$ and associated with innate immunity against viral infections. ${ }^{18}$ However, little is known about its function in the endometrium and endometriotic lesions. Although the significance of IFITM1 expression in the endometrium remains unclear, it is reasonable to assume that it is associated with the local innate 


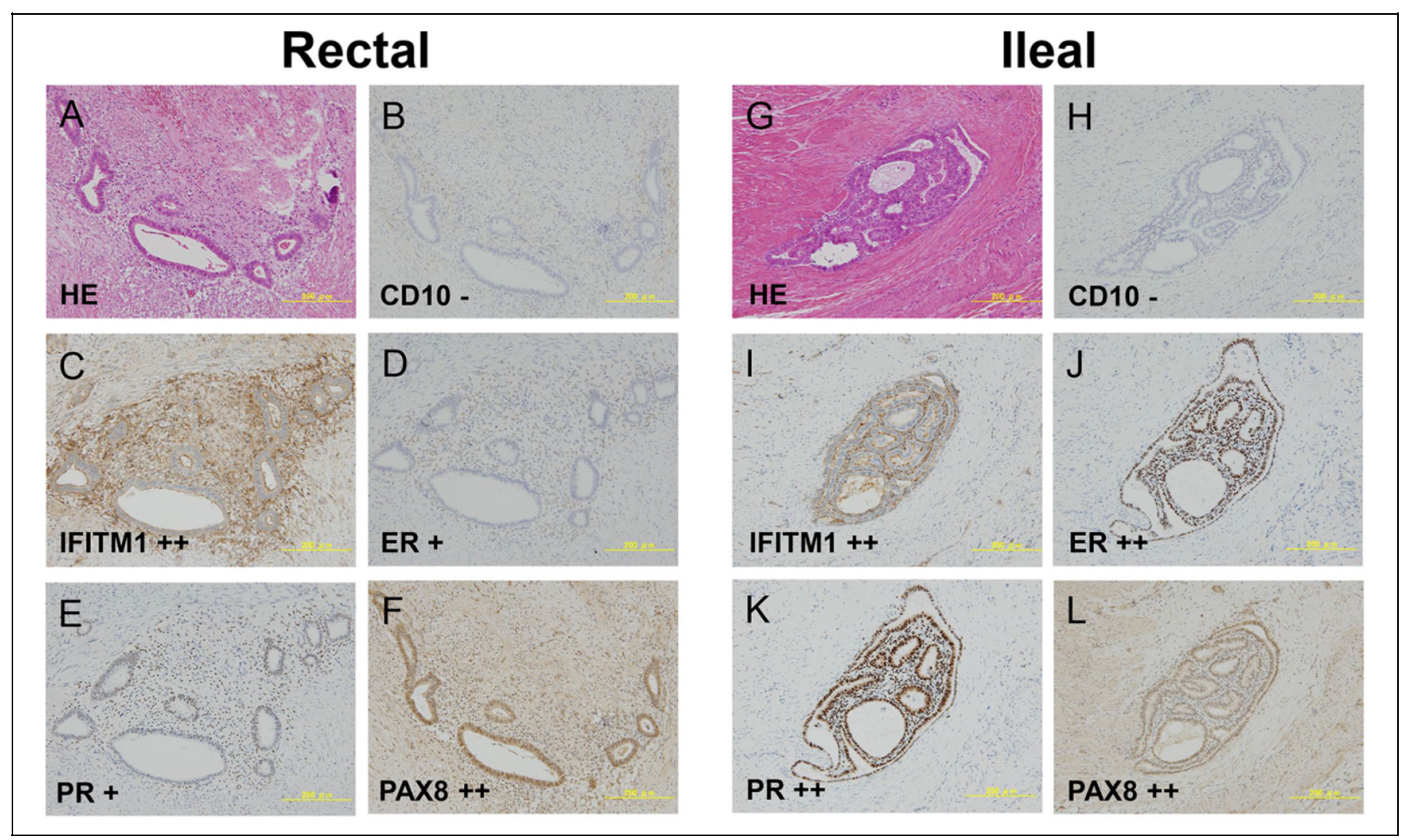

Figure 4. CDI0-negative and interferon-inducible transmembrane protein I (IFITMI)-, ER-, progesterone receptor (PR)-, and PAX8-positive expression in extragenital endometriosis. ( $A$ and $G) H \& E$ and $(B$ and $H) C D I 0$-negative expression in cases of rectal and ileal endometriosis. Rectal endometriosis: (C) IFITMI staining was diffuse in stromal cells, (D) estrogen receptor (ER) and (E) PR staining was positive in stromal cells, and (F) PAX8 staining was positive in the nucleus of epithelial cells. Ileal endometriosis: (I) IFITMI staining was diffuse in stromal cells, (J) ER and $(K)$ PR staining was positive in stromal and epithelial cells, and L, PAX8 staining was positive in the nucleus of epithelial cells. Magnification, $\times 200$. - , negative; + , positive; ++ , strongly positive.

Table 3. The Comparison of IFITMI and CDIO Expression in Extragenital Endometriosis With or Without Hormonal Treatment.

\begin{tabular}{lcccccc}
\hline Marker & Without Hormonal Treatment & With Hormone Treatment & $P$ Value & GnRH Agonist & Dienogest & Oral Contraceptives \\
\hline IFITMI & $39 / 39(100.0 \%)$ & $23 / 23(100.0 \%)$ & NS & $16 / 16(100.0 \%)$ & $5 / 5(100.0 \%)$ & $2 / 2(100.0 \%)$ \\
CDI0 & $37 / 39(94.9 \%)$ & $23 / 23(100.0 \%)$ & NS & $16 / 16(100.0 \%)$ & $5 / 5(100.0 \%)$ & $2 / 2(100.0 \%)$ \\
\hline
\end{tabular}

Abbreviations: GnRH, gonadotropin-releasing hormone; IFITMI, Interferon-inducible transmembrane protein I.

immune system in this area. In cancer cells, IFITM1 is associated with cell invasion and metastasis, ${ }^{21-23}$ suggesting a potential link with endometriosis pathophysiology. Further studies are required to clarify for the role of IFITM1 in the endometrium and endometriosis.

In summary, we demonstrated that IFITM1 is a highly sensitive stromal marker of endometriosis and extragenital endometriosis. Given our findings showing IFITM1 sensitivity as comparable or possibly superior to that of CD10, IFITM1 can be a useful addition in immunohistochemical examination of the disease, particularly in the accurate diagnosis of endometriosis in cases of ambiguous or unexpected CD10 expression. The efficacy of IFITM1 in this context should be validated by further examination and in clinical practice.

\section{Authors' Note}

Hui Sun and Shinya Fukuda considered that the first 2 authors should be regarded as joint first authors.

\section{Acknowledgments}

The authors would like to thank Editage (www.editage.jp) for the English-language review.

\section{Declaration of Conflicting Interests}

The author(s) declared no potential conflicts of interest with respect to the research, authorship, and/or publication of this article.

\section{Funding}

The author(s) disclosed receipt of the following financial support for the research, authorship, and/or publication of this article: This work 
was supported by grants from the Ministry of Health, Labour and Welfare, the Ministry of Education, Culture, Sports, Science and Technology and the Japan Agency for Medical Research and Development.

\section{ORCID iD}

Tetsuya Hirata, MD, PhD (D) https://orcid.org/0000-0002-9975-6208

\section{References}

1. Zondervan KT, Becker CM, Koga K, Missmer SA, Taylor RN, Vigano P. Endometriosis. Nat Rev Dis Primers. 2018;4(1):9.

2. Saito A, Koga K, Osuga Y, et al. Individualized management of umbilical endometriosis: a report of seven cases. J Obstet Gynaecol Res. 2014;40(1):40-45.

3. Fukuda S, Hirata T, Neriishi K, et al. Thoracic endometriosis syndrome: Comparison between catamenial pneumothorax or endometriosis-related pneumothorax and catamenial hemoptysis. Eur J Obstet Gynecol Reprod Biol. 2018;225:118-123.

4. Groisman GM, Meir A. CD10 is helpful in detecting occult or inconspicuous endometrial stromal cells in cases of presumptive endometriosis. Arch Pathol Lab Med. 2003;127(8):1003-1006.

5. Oliva E. CD10 expression in the female genital tract: does it have useful diagnostic applications? Adv Anat Pathol. 2004;11(6): 310-315.

6. Potlog-Nahari C, Feldman AL, Stratton P, et al. CD10 immunohistochemical staining enhances the histological detection of endometriosis. Fertil Steril. 2004;82(1):86-92.

7. Sumathi VP, McCluggage WG. CD10 is useful in demonstrating endometrial stroma at ectopic sites and in confirming a diagnosis of endometriosis. J Clin Pathol. 2002;55(5):391-392.

8. Vigano P, Candiani M, Monno A, Giacomini E, Vercellini P, Somigliana E. Time to redefine endometriosis including its profibrotic nature. Hum Reprod. 2018;33(3):347-352.

9. Chu P, Arber DA. Paraffin-section detection of CD10 in 505 nonhematopoietic neoplasms. Frequent expression in renal cell carcinoma and endometrial stromal sarcoma. Am J Clin Pathol. 2000;113(3):374-382.

10. Parra-Herran CE, Yuan L, Nucci MR, Quade BJ. Targeted development of specific biomarkers of endometrial stromal cell differentiation using bioinformatics: the IFITM1 model. Mod Pathol. 2014;27(4):569-579.

11. Abeler VM, Nenodovic M. Diagnostic immunohistochemistry in uterine sarcomas: a study of 397 cases. Int J Gynecol Pathol. 2011;30(3):236-243.
12. D'Angelo E, Spagnoli LG, Prat J. Comparative clinicopathologic and immunohistochemical analysis of uterine sarcomas diagnosed using the World Health Organization classification system. Hum Pathol. 2009;40(11):1571-1585.

13. Busca A, Djordjevic B, Giassi A, Parra-Herran C. IFITM1 Is Superior to CD10 as a marker of endometrial stroma in the evaluation of myometrial invasion by endometrioid adenocarcinoma. Am J Clin Pathol. 2016;145(4):486-496.

14. Busca A, Gulavita P, Parra-Herran C, Islam S. IFITM1 Outperforms CD10 in differentiating low-grade endometrial stromal sarcomas from smooth muscle neoplasms of the uterus. Int J Gynecol Pathol. 2018;37(4):372-378.

15. McCluggage WG, Sumathi VP, Maxwell P. CD10 is a sensitive and diagnostically useful immunohistochemical marker of normal endometrial stroma and of endometrial stromal neoplasms. Histopathology. 2001;39(3):273-278.

16. Mikami Y, Hata S, Kiyokawa T, Manabe T. Expression of CD10 in malignant mullerian mixed tumors and adenosarcomas: an immunohistochemical study. Mod Pathol. 2002;15(9): 923-930

17. Toki T, Shimizu M, Takagi Y, Ashida T, Konishi I. CD10 is a marker for normal and neoplastic endometrial stromal cells. Int $J$ Gynecol Pathol. 2002;21(1):41-47.

18. Bailey CC, Zhong G, Huang IC, Farzan M. IFITM-family proteins: the cell's first line of antiviral defense. Annu Rev Virol. 2014;1:261-283.

19. Friedman RL, Manly SP, McMahon M, Kerr IM, Stark GR. Transcriptional and posttranscriptional regulation of interferoninduced gene expression in human cells. Cell. 1984;38(3): 745-755.

20. Reid LE, Brasnett AH, Gilbert CS, et al. A single DNA response element can confer inducibility by both alpha- and gamma-interferons. Proc Natl Acad Sci U S A. 1989;86(3):840-844.

21. Hatano H, Kudo Y, Ogawa I, et al. IFN-induced transmembrane protein 1 promotes invasion at early stage of head and neck cancer progression. Clin Cancer Res. 2008;14(19):6097-6105.

22. Yu F, Ng SS, Chow BK, et al. Knockdown of interferon-induced transmembrane protein 1 (IFITM1) inhibits proliferation, migration, and invasion of glioma cells. J Neurooncol. 2011;103(2): 187-195.

23. Yu F, Xie D, Ng SS, et al. IFITM1 promotes the metastasis of human colorectal cancer via CAV-1. Cancer Lett. 2015;368(1): 135-143. 